\title{
Generalized Weighted Exponential-Gompertez Distribution
}

\author{
Abd El-Moneim A. M. Teamah, Hanan H. El-Damrawy, Shaimaa M. T. Swan \\ Mathematics Department, Faculty of Science, Tanta University, Tanta, Egypt \\ Email: abdelmoneam.toeama@science.tanta.edu.eg, hanan.eldamarawi@science.tanta.edu.eg, shimaa.swan@gmail.com
}

How to cite this paper: Teamah, A.A.M., El-Damrawy, H.H. and Swan, S.M.T. (2020) Generalized Weighted ExponentialGompertez Distribution. Applied Mathematics, 11, 97-118. https://doi.org/10.4236/am.2020.112010

Received: December 2, 2019

Accepted: February 17, 2020

Published: February 20, 2020

Copyright (c) 2020 by author(s) and Scientific Research Publishing Inc. This work is licensed under the Creative Commons Attribution International License (CC BY 4.0).

http://creativecommons.org/licenses/by/4.0/

\section{(c) (i) Open Access}

\begin{abstract}
Statistical analysis of lifetime data is a significant topic in social sciences, engineering, reliability, biomedical and others. We use the generalized weighted exponential distribution, as a generator to introduce a new family called generalized weighted exponential-G family, and apply this new generator to provide a new distribution called generalized weighted exponential gombertez distribution. We investigate some of its properties, moment generating function, moments, conditional moments, mean residual lifetime, mean inactivity time, strong mean inactivity time, Rényi entropy, Lorenz curves and Bonferroni. Furthermore, in this model, we estimate the parameters by using maximum likelihood method. We apply this model to a real data-set to show that the new generated distribution can produce a better fit than other classical lifetime models.
\end{abstract}

\section{Keywords}

Generalized Weighted Exponential-G Family, Gombertez Distribution, Bonferroni and Lorenz Curves, Conditional Moments, Mean Inactivity Time, Mean Residual Lifetime, Maximum Likelihood Estimators

\section{Introduction}

Generally, there is a rising interest in the introduction of new generators for univariate continuous families of distributions by adding one or more additional shape parameter(s) to the baseline distribution. This introduction of parameter(s) was proven useful in the exploration of tail properties and the improvement of the goodness-of-fit of the family under investigation. There are well-known generators like the following: the beta-G distribution by Eugene et al. [1], the gamma-G type 1 distribution by Zografos and Balakrishanan [2] and Amini et al. [3], the Kumaraswamy-G (Kw-G) distribution by Cordeiro and de 
Castro [4], the McDonald-G (Mc-G) distribution by Alexander et al. [5], the gamma-G type 2 distribution by Ristic and Balakrishanan [6] and Amini et al. [3], the odd exponentiated generalized (odd exp-G) distribution by Cordeiro et al. [7], the transformed transformer (T-X) (Weibull-X and gamma-X) distribution by Alzaatreh et al. [8], the odd Weibull-G distribution by Bourguignon et al. [9], the Lomax-G distribution by Cordeiro et al. [10], the Kumaraswamy odd log-logistic-G distribution by Alizadeh et al. [11] and the logistic-X distribution by Tahir et al. [12], a new family by using survival function called weighted exponential-G family introduced by Abd El-Bar et al. [13].

The class of weighted exponential (WE) distributions was obtained by Gupta and Kundu [14] by implementing Azzalini's method to the exponential distribution. Shakhatreh [15] generalized the WE distribution to the two-parameter weighted exponential distributions (TWE). Finally, Kharazmi et al. [16] proposed a generalized weighted exponential distribution (GWE). It is observed that the WE, TWE and GWE distribution can provide a better fit for survival time data relative to other common distributions such as gamma, Weibull, or generalized exponential distribution.

The aim of this paper is to introduce a new family of distributions generated by generalized weighted exponential (GWE) distribution. We expect that the proposed family may be better (at least in terms of model fitting) than other classes of distributions in certain practical situations. The proposed GWE-G distribution provides WE-G and TWE-G distributions as its sub-models. This extension offers more flexible distributions with applications in reliability engineering and lifetime modeling. In addition, several properties of the GWE-G distribution have been established.

In the following sections, we study the properties of a special case of this family, when $G(\cdot)$ is the CDF of the Gompertz distribution. In this case, the random variable $\mathrm{X}$ is said to have the generalized weighted exponential-Gompertz (GWE-G) distribution.

The reminder of this paper is organized as follows. We discuss the generalized weighted exponential-G family in Section 2. In Section 3, the generalized weighted exponential-Gompertz distribution is studied in detail. In Section 4, we provide expansions for weighted exponential-Gompertz cumulative and density functions. In Section 5, we present various properties of the new model such as moment generating function, moments and conditional moments. Rényi Entropy introduced in Section 6. Also, some reliability properties of our model are discussed in Section 7. In Section 8, the maximum likelihood estimator of the parameters of our model is obtained. Section 9 gives an application to a real data set.

\section{Generalized Weighted Exponential-G Family}

Ristić and Balakrishnan [6] introduced a new family of distributions generated by gamma random variable with survival function (SF) given by: 


$$
\bar{F}(x)=\frac{1}{\Gamma(\delta)} \int_{0}^{-\log G(x)} t^{\delta-1} \mathrm{e}^{-t} \mathrm{~d} t, \quad x \in R, \delta>0
$$

where $G(x)$ is a cumulative distribution function (CDF) of baseline distribution which is used to generate a new family of continuous distributions. On the same line, we provide a new family of distributions generated by generalized exponential distribution.

If a random variable $\mathrm{X}$ has the generalized weighted exponential distribution GWE $(\beta, \alpha, n)$ with integer $n \geq 1$, shape parameter $\alpha$ and scale parameter $\beta$, if its probability density function(PDF) is given by:

$$
f(x, \beta, \alpha, n)=\frac{\alpha}{B\left(\frac{1}{\alpha}, n+1\right)} \beta \mathrm{e}^{-\beta x}\left(1-\mathrm{e}^{-\alpha \beta x}\right)^{n}, \quad x, \alpha, \beta>0
$$

where, $B(a, b)=\int_{0}^{1} t^{a-1}(1-t)^{b-1} \mathrm{~d} t$, and the corresponding CDF is given by:

$$
F(x, \beta, \alpha, n)=1-\frac{\alpha}{B\left(\frac{1}{\alpha}, n+1\right)} \sum_{k=0}^{n} \frac{(-1)^{k}\left(\begin{array}{l}
n \\
k
\end{array}\right)}{\alpha k+1} \mathrm{e}^{-\beta(\alpha k+1) x} .
$$

Now, by using an integral transform of the PDF of a random variable $\mathrm{T}$ which follows GWE distribution we generate a new family of distributions. The SF of generalized weighted exponential-G (GWE-G) family is defined as:

$$
\begin{aligned}
\bar{F}(x, \alpha, \beta, \xi) & =\frac{\alpha \beta}{B\left(\frac{1}{\alpha}, n+1\right)} \int_{0}^{-\log G(x, \xi)} \mathrm{e}^{-\beta x}\left(1-\mathrm{e}^{-\alpha \beta x}\right)^{n} \mathrm{~d} x \\
& =\frac{\alpha}{B\left(\frac{1}{\alpha}, n+1\right)} \sum_{i=0}^{n} \frac{(-1)^{i}\left(\begin{array}{c}
n \\
i
\end{array}\right)}{\alpha i+1}\left(1-G^{\beta(\alpha i+1)}(x)\right) \\
& =1-\frac{\alpha}{B\left(\frac{1}{\alpha}, n+1\right)} \sum_{i=0}^{n} \frac{(-1)^{i}\left(\begin{array}{l}
n \\
i
\end{array}\right)}{\alpha i+1} G^{\beta(\alpha i+1)}(x) .
\end{aligned}
$$

and the PDF of GWE-G family is obtained by:

$$
\begin{aligned}
f(x, \alpha, \beta, \xi) & =\frac{\alpha \beta}{B\left(\frac{1}{\alpha}, n+1\right)} \sum_{i=0}^{n}(-1)^{i}\left(\begin{array}{l}
n \\
i
\end{array}\right) g(x) G^{\beta(\alpha i+1)-1}(x) \\
& =\frac{\alpha \beta}{B\left(\frac{1}{\alpha}, n+1\right)} g(x) G^{\beta-1}(x)\left[1-G^{\alpha \beta}(x)\right]^{n}
\end{aligned}
$$

where, $G(x, \xi)$ and $g(x, \xi)$ respectively, are the baseline CDF and PDF which depends on a $(p \times 1)$ parameter vector $\xi$ and $(\alpha, \beta)$ are two additional parameters. 


\section{Generalized Weighted Exponential-Gompertz Distribution}

The PDF of GD with location parameter $\sigma$ and shape parameter $\lambda$, is given by:

$$
g(x, \lambda, \sigma)=\lambda \sigma \exp \left[\lambda x-\sigma\left(\mathrm{e}^{\lambda x}-1\right)\right]
$$

The CDF associated with Equation (6) is obtained as:

$$
G(x, \lambda, \sigma)=1-\exp \left[-\sigma\left(\mathrm{e}^{\lambda x}-1\right)\right]
$$

The GD plays an important role in modeling reliability, human mortality and actuarial data that have hazard rate with exponential increase. Some applications of this distribution can be found in Pollard and Valkovics [17]. An extension version of Gompertz is the weighted Gompertz distribution discussed by Bakouch and Abd El-Bar [18].

Now, we introduce the PDF and CDF of GWE-G distribution by using Equations (4)-(6) respectively, are:

$$
\begin{aligned}
f(x, \alpha, \beta, \lambda, \sigma, n) & =\frac{\alpha \beta \lambda \sigma}{B\left(\frac{1}{\alpha}, n+1\right)} \sum_{i=0}^{n}(-1)^{i}\left(\begin{array}{l}
n \\
i
\end{array}\right) \mathrm{e}^{\lambda x} A^{\beta(\alpha i+1)-1}(x)[1-A(x)] \\
& =\frac{\alpha \beta \lambda \sigma}{B\left(\frac{1}{\alpha}, n+1\right)} \mathrm{e}^{\lambda x} A^{\beta-1}(x)[1-A(x)]\left[1-A^{\alpha \beta}(x)\right]^{n} .
\end{aligned}
$$

and,

$$
F(x, \alpha, \beta, \lambda, \sigma, n)=\frac{\alpha}{B\left(\frac{1}{\alpha}, n+1\right)} \sum_{i=0}^{n} \frac{(-1)^{i}\left(\begin{array}{l}
n \\
i
\end{array}\right)}{\alpha i+1} A^{\beta(\alpha i+1)}(x)
$$

where, $A(x)=1-\exp \left[-\sigma\left(\mathrm{e}^{\lambda x}-1\right)\right]$.

Figure 1 shows the shapes of GWE-G distribution or some various parameters. It can be summarized some of the shape properties of our model as: (a) The PDF is left-skewed when $(\alpha=2, \beta=1, \lambda=2, \sigma=0.03)$ and $n=1,2,5$ and 7. (b) The PDF is right-skewed when $(\alpha=1, \sigma=3, n=1)$ and $\beta>1$ and $\lambda>1$ and the PDF is reserved-J when $(\alpha=1, \sigma=3, n=1)$ and $\beta>1$ and $\lambda<1$. (c) The $\mathrm{PDF}$ is monotonically decreasing when $(\beta=2, \lambda=1, n=1)$ and $\alpha<1$ and $\sigma<1$. (d) The PDF is reserved-J when $(\beta=2, \lambda=1, n=1)$ and $\alpha<1$ and $\sigma \geq 1$. (e) The PDF is left-skewed when $(\beta=2, \lambda=1, n=1)$ and $\alpha \geq 1$ and $\sigma<1$. (f) The PDF is left-skewed when $(\beta=1, \lambda=2, n=1)$ and $\alpha \geq 1$ and $\sigma>1$.

The SF and HR of the GWE-G distribution respectively, are:

$$
S(x, \alpha, \beta, \lambda, \sigma, n)=\frac{\alpha}{B\left(\frac{1}{\alpha}, n+1\right)} \sum_{i=0}^{n} \frac{(-1)^{i}\left(\begin{array}{c}
n \\
i
\end{array}\right)}{\alpha i+1}\left[1-A^{\beta(\alpha i+1)}(x)\right]=1-\frac{\alpha}{B\left(\frac{1}{\alpha}, n+1\right)} \sum_{i=0}^{n} \frac{(-1)^{i}\left(\begin{array}{c}
n \\
i
\end{array}\right)}{\alpha i+1} A^{\beta(\alpha i+1)}(x)
$$

and, 


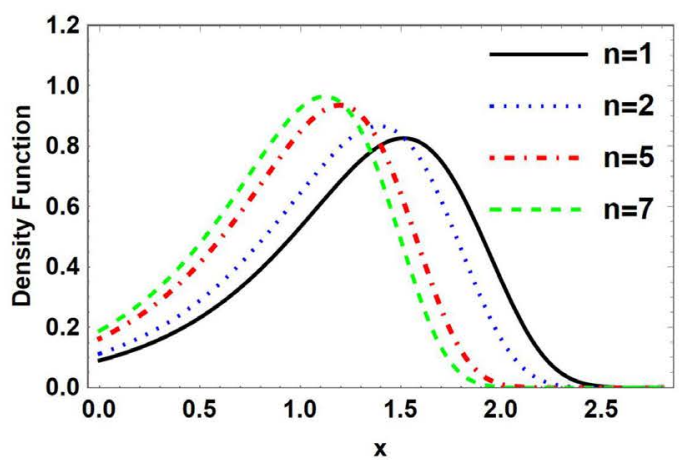

(a)

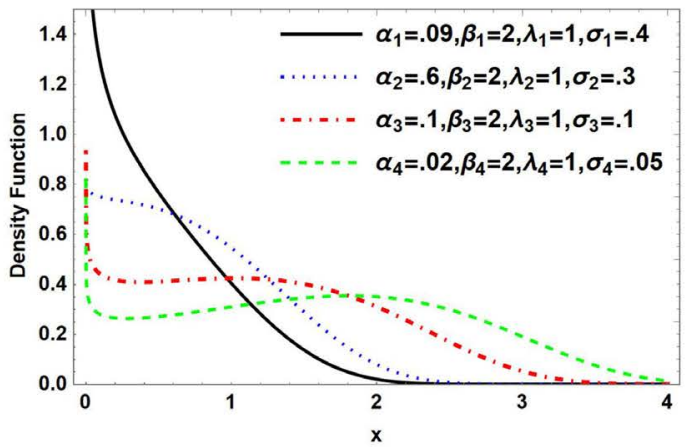

(c)

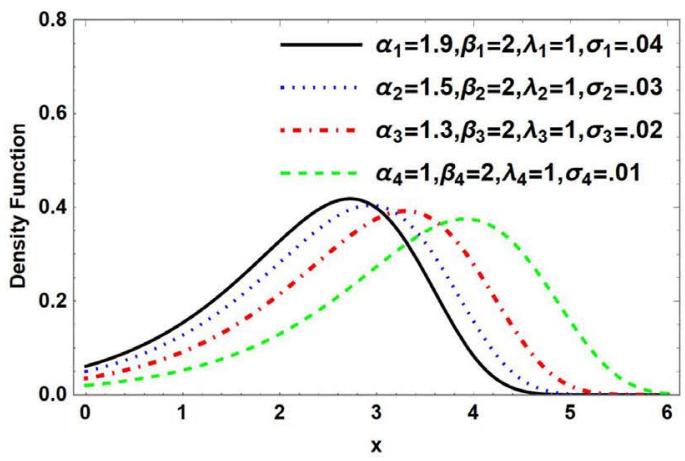

(e)

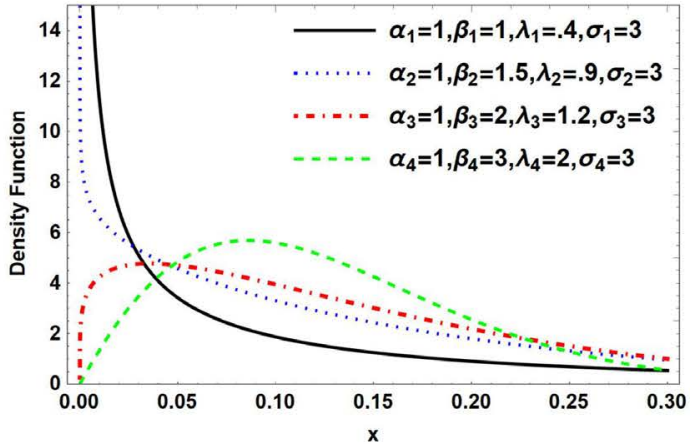

(b)

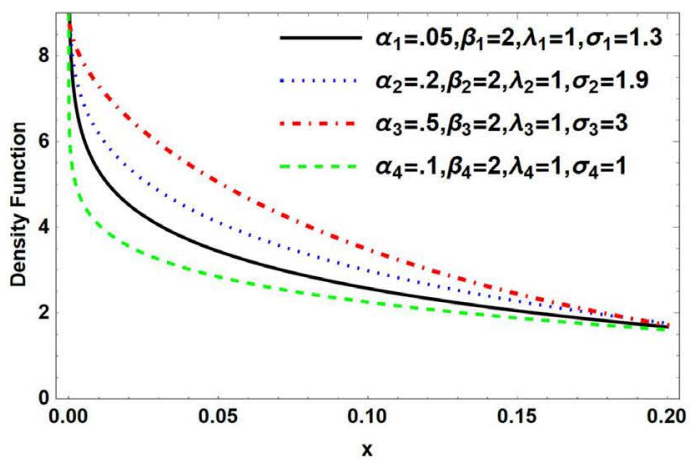

(d)

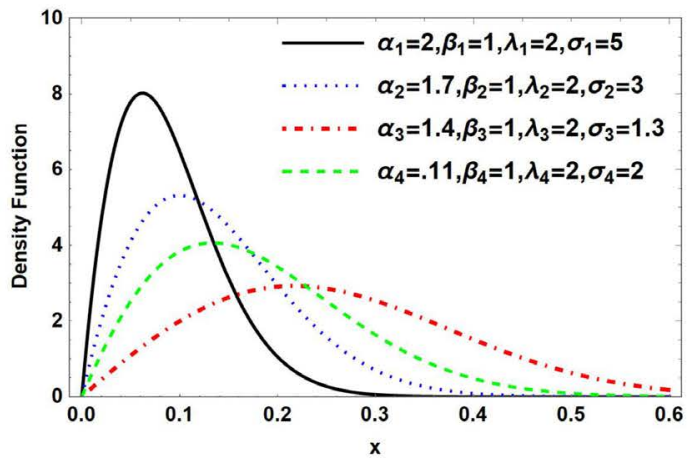

(f)

Figure 1. The PDF curves of GWE-G with $(\beta, \alpha, \lambda, \sigma, n)$.

$$
h(x, \alpha, \beta, \lambda, \sigma, n)=\frac{\beta \lambda \sigma \sum_{i=0}^{n}(-1)^{i}\left(\begin{array}{c}
n \\
i
\end{array}\right) \mathrm{e}^{\lambda x} A^{\beta(\alpha i+1)-1}(x)[1-A(x)]}{\sum_{i=0}^{n} \frac{(-1)^{i}\left(\begin{array}{c}
n \\
i
\end{array}\right)}{\alpha i+1}\left[1-A^{\beta(\alpha i+1)}(x)\right]} .
$$

Figure 2 gives some of the possible shapes of the hazard rate function of the GWE-G distribution for some various values of parameters $\mathrm{n}, \alpha, \beta, \lambda$ and $\sigma$. It can be summarized some of the shape properties of the hazard rate function of 


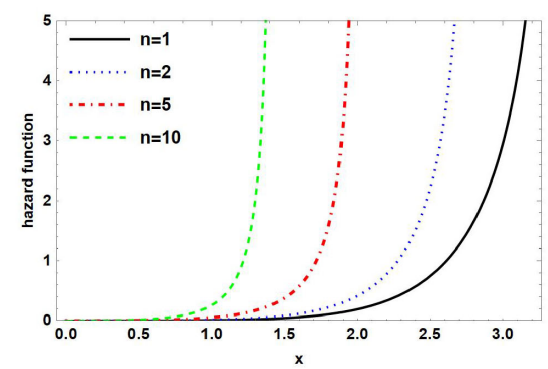

(a)

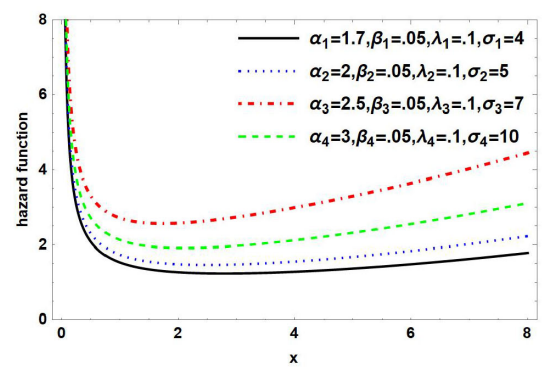

(b)

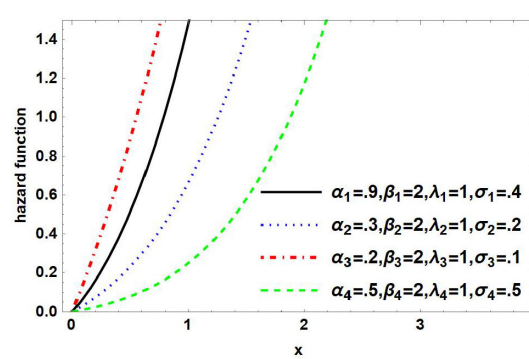

(c)

Figure 2. The hazard curves of GWE-G with $(\alpha, \beta, \lambda, \sigma, n)$.

WE-G as: (a) The hazard rate function is increasing function when $(\alpha=0.5, \beta=3, \lambda=1, \sigma=0.3)$ and $n=1,2,5$ and 10. (b) The hazard rate function is bathtub shaped when $(\beta=0.05, \lambda=0.1, n=1)$ and $\alpha \geq 1$ and $\sigma \geq 1$. (c) The hazard rate function is increasing function when $(\beta=2, \lambda=1, n=1)$ and $\alpha<1$ and $\sigma<1$.

\section{Expansions for the Cumulative, Density and Survival Function}

In this section, we discuss some useful expansions for the CDF and PDF of GWE-G distribution. The following mathematical relations will be used in these expansions.

$$
(1-z)^{k}=\sum_{j=0}^{\infty}(-1)^{j}\left(\begin{array}{l}
k \\
j
\end{array}\right) z^{j},
$$

for any positive real non-integer $k$, and $|Z|<1$. We also use this relation for any positive integer $m$ :

$$
(a-b)^{m}=\sum_{j=0}^{m}(-1)^{j}\left(\begin{array}{c}
m \\
j
\end{array}\right) b^{j} a^{m-j}
$$

\subsection{Expansion for the CDF}

In this subsection, we introduce the expansion forms for the CDF for GWE-G $(\alpha, \beta, \lambda, \sigma, n)$ distribution. We can writ Equation (9) in another form:

$$
\begin{aligned}
F(x, \alpha, \beta, \lambda, \sigma, n) & =\frac{\alpha}{B\left(\frac{1}{\alpha}, n+1\right)} \sum_{i=0}^{n} \frac{(-1)^{i}\left(\begin{array}{l}
n \\
i
\end{array}\right)}{\alpha i+1}\left[1-\exp ^{-\sigma\left(\mathrm{e}^{\lambda x}-1\right)}\right]^{\beta(\alpha i+1)-1} \\
& =\sum_{i=0}^{n} \varepsilon_{i} G(x, \sigma, \lambda, \beta(\alpha i+1)-1) .
\end{aligned}
$$

where, $\varepsilon_{i}=\frac{\alpha(-1)^{i}\left(\begin{array}{l}n \\ i\end{array}\right)}{(\alpha i+1) B\left(\frac{1}{\alpha}, n+1\right)}$, and $G(x, \sigma, \lambda, \beta(\alpha i+1)-1)$ denotes the ex-

ponentiated Gompertz (EG) distribution with parameters $\sigma, \lambda$ and 
$\beta(\alpha i+1)-1$.

We also obtain another expansion for the CDF of GWE-G as:

From Equation (9) and expanding the term $\left[1-\exp ^{-\sigma\left(\mathrm{e}^{\lambda x}-1\right)}\right]^{\beta(\alpha i+1)-1}$, the GWE-G can be rewritten as:

$$
\begin{aligned}
& F(x, \alpha, \beta, \lambda, \sigma, n) \\
& =\sum_{i=0}^{n} \sum_{j=0}^{\infty} \frac{\alpha(-1)^{i+j}}{(\alpha i+1) B\left(\frac{1}{\alpha}, n+1\right)}\left(\begin{array}{c}
n \\
i
\end{array}\right)\left(\begin{array}{c}
\beta(\alpha i+1)-1 \\
j
\end{array}\right) \exp ^{-\sigma j\left(e^{\lambda x}-1\right)}
\end{aligned}
$$

Using power series expansion for $\left(\exp \left[-\sigma j\left(\mathrm{e}^{\lambda x}-1\right)\right]\right)$, we have:

$$
\begin{aligned}
& F(x, \alpha, \beta, \lambda, \sigma, n) \\
& =\sum_{i=0}^{n} \sum_{j, k=0}^{\infty} \frac{\alpha(-1)^{i+j+k}}{(\alpha i+1) B\left(\frac{1}{\alpha}, n+1\right)}\left(\begin{array}{c}
n \\
i
\end{array}\right)\left(\begin{array}{c}
\beta(\alpha i+1)-1 \\
j
\end{array}\right) \frac{(\sigma j)^{k}}{k !}\left[\left(\mathrm{e}^{\lambda x}-1\right)^{k}\right]
\end{aligned}
$$

Now, using the binomial expansion for $\left(\mathrm{e}^{\lambda x}-1\right)^{k}$, the CDF admits the following expansion:

$$
\begin{aligned}
& F(x, \alpha, \beta, \lambda, \sigma, n) \\
& =\sum_{i=0}^{n} \sum_{j, k=0 l=0}^{\infty} \sum_{(\alpha i+1) B\left(\frac{1}{\alpha}, n+1\right)}^{k}\left(\begin{array}{l}
n \\
i
\end{array}\right)\left(\begin{array}{c}
k \\
l
\end{array}\right)\left(\begin{array}{c}
\beta(\alpha i+1)-1 \\
j
\end{array}\right) \frac{(\sigma j)^{k}}{k !}\left(\mathrm{e}^{\lambda x(k-l)}\right)
\end{aligned}
$$

Also, the GWE-G CDF can be rewritten as:

$$
\begin{aligned}
& F(x, \alpha, \beta, \lambda, \sigma, n) \\
& =\sum_{i=0}^{n} \sum_{j, k, m=0}^{\infty} \sum_{l=0}^{k} \frac{\alpha(\sigma j)^{k}(\lambda)^{m}(k-l)^{m}(-1)^{i+j+k+l}}{(\alpha i+1) k ! m ! B\left(\frac{1}{\alpha}, n+1\right)}\left(\begin{array}{c}
n \\
i
\end{array}\right)\left(\begin{array}{c}
k \\
l
\end{array}\right)\left(\begin{array}{c}
\beta(\alpha i+1)-1 \\
j
\end{array}\right) x^{m} \\
& =\sum_{i=0}^{n} \sum_{m, k, j=0}^{\infty} \sum_{l=0}^{k} \rho_{i, j, k, l, m} x^{m}
\end{aligned}
$$

where,

$$
\rho_{i, j, k, l, m}=(-1)^{i+j+k+l}\left(\begin{array}{c}
\beta(\alpha i+1)-1 \\
j
\end{array}\right)\left(\begin{array}{l}
n \\
i
\end{array}\right)\left(\begin{array}{l}
k \\
l
\end{array}\right) \frac{\alpha \lambda^{m}(\sigma j)^{k}(k-l)^{m}}{(\alpha i+1) k ! m ! B\left(\frac{1}{\alpha}, n+1\right)} .
$$

\subsection{Expansion for the SF}

In this subsection, we introduce the expansion forms for the SF for GWE-G $(\alpha, \beta, \lambda, \sigma, n)$ distribution. From Equation (10) and using Equation (13), the SF of GWE-G can be rewritten as:

$$
S(x, \alpha, \beta, \lambda, \sigma, n)=\frac{\alpha}{B\left(\frac{1}{\alpha}, n+1\right)} \sum_{i=0}^{n} \sum_{j=0}^{1} \frac{(-1)^{i+j}\left(\begin{array}{l}
n \\
i
\end{array}\right)}{\alpha i+1} A^{\beta j(\alpha i+1)}(x) .
$$


expanding the term $\left[1-\exp ^{-\sigma\left(\mathrm{e}^{\lambda x}-1\right)}\right]^{\beta j(\alpha i+1)-1}$, we have:

$$
\begin{aligned}
& S(x, \alpha, \beta, \lambda, \sigma, n) \\
& =\sum_{i=0}^{n} \sum_{j=0}^{1} \sum_{k=0}^{\infty} \frac{\alpha(-1)^{i+j+k}}{(\alpha i+1) B\left(\frac{1}{\alpha}, n+1\right)}\left(\begin{array}{c}
n \\
i
\end{array}\right)\left(\begin{array}{c}
\beta j(\alpha i+1)-1 \\
k
\end{array}\right)\left[\exp ^{-\sigma k\left(e^{\lambda x}-1\right)}\right]
\end{aligned}
$$

Using power series expansion for $\left[\exp ^{-\sigma k\left(\mathrm{e}^{2 x}-1\right)}\right]$, we have:

$$
\begin{aligned}
& S(x, \alpha, \beta, \lambda, \sigma, n) \\
& =\sum_{i=0}^{n} \sum_{j=0 k, l=0}^{1} \sum_{(\alpha i+1) B\left(\frac{1}{\alpha}, n+1\right)}^{\infty}\left(\begin{array}{c}
n \\
i
\end{array}\right)\left(\begin{array}{c}
\beta j(\alpha i+1)-1 \\
k
\end{array}\right) \frac{(\sigma k)^{l}}{l !}\left[\left(\mathrm{e}^{\lambda x}-1\right)^{l}\right]
\end{aligned}
$$

Now, using the binomial expansion for $\left(\mathrm{e}^{\lambda x}-1\right)^{l}$, the CDF admits the following expansion:

$$
\begin{aligned}
& S(x, \alpha, \beta, \lambda, \sigma, b) \\
& =\sum_{i=0}^{n} \sum_{j=0 k, l=0}^{1} \sum_{m=0}^{\infty} \frac{\alpha(-1)^{i+j+k+l+m}}{(\alpha i+1) B\left(\frac{1}{\alpha}, n+1\right)}\left(\begin{array}{c}
n \\
i
\end{array}\right)\left(\begin{array}{c}
l \\
m
\end{array}\right)\left(\begin{array}{c}
\beta j(\alpha i+1)-1 \\
k
\end{array}\right) \frac{(\sigma k)^{l}}{l !}\left(\mathrm{e}^{\lambda x(l-m)}\right) \\
& =\sum_{i=0}^{n} \sum_{j=0}^{1} \sum_{k, l=0}^{\infty} \sum_{m=0}^{l} v_{i, j, k, l, m}\left(\mathrm{e}^{\lambda x(l-m)}\right)
\end{aligned}
$$

where,

$$
v_{i, j, k, l, m}=\frac{\alpha(-1)^{i+j+k+l+m}}{(\alpha i+1) B\left(\frac{1}{\alpha}, n+1\right)}\left(\begin{array}{l}
n \\
i
\end{array}\right)\left(\begin{array}{l}
l \\
m
\end{array}\right)\left(\begin{array}{c}
\beta j(\alpha i+1)-1 \\
k
\end{array}\right) \frac{(\sigma k)^{l}}{l !}
$$

\subsection{Expansion for the PDF}

Here, we provide simple expansion for the GWE-G density function. Firstly, applying Equation (13) into Equation (8) and expansion the term:

$$
\begin{aligned}
& {\left[1-\exp ^{-\sigma\left(\mathrm{e}^{\lambda x}-1\right)}\right]^{\beta(\alpha i+1)+j-1}, \text { yields: }} \\
& \quad f(x, \alpha, \beta, \lambda, \sigma, n) \\
& \quad=\frac{\alpha \beta \lambda \sigma \mathrm{e}^{\lambda x}}{B\left(\frac{1}{\alpha}, n+1\right)} \sum_{i=0}^{n} \sum_{j=0 k, l=0}^{1} \sum^{\infty}(-1)^{i+j+k+l}\left(\begin{array}{c}
n \\
i
\end{array}\right)\left(\begin{array}{c}
\beta(\alpha i+1)+j-1 \\
k
\end{array}\right) \frac{(\sigma k)^{l}}{l !}\left[\left(\mathrm{e}^{\lambda x}-1\right)^{l}\right] .
\end{aligned}
$$

Using again the series expansion (13), we can express the PDF of GWE-G distribution as:

$$
\begin{aligned}
& f(x, \alpha, \beta, \lambda, \sigma, n) \\
& =\frac{\alpha \beta \lambda}{B\left(\frac{1}{\alpha}, n+1\right)} \sum_{i=0}^{n} \sum_{j=0}^{1} \sum_{k, l=0}^{\infty} \sum_{m=0}^{l}(-1)^{i+j+k+l+m}\left(\begin{array}{c}
\beta(\alpha i+1)+j-1 \\
k
\end{array}\right) \times\left(\begin{array}{c}
n \\
i
\end{array}\right)\left(\begin{array}{c}
l \\
m
\end{array}\right) \frac{(\sigma)^{l+1} k^{l}}{l !}\left[\mathrm{e}^{\lambda x(l-m+1)}\right]
\end{aligned}
$$




$$
=\sum_{i=0}^{n} \sum_{j=0 k, l=0}^{1} \sum_{m=0}^{\infty} \sum_{i, j, k, l, m}^{l}\left[\mathrm{e}^{\lambda x(l-m+1)}\right] .
$$

where,

$$
\phi_{i, j, k, l, m}=\frac{\alpha \beta \lambda(\sigma)^{l+1} k^{l}(-1)^{i+j+k+l+m}}{l ! B\left(\frac{1}{\alpha}, n+1\right)}\left(\begin{array}{c}
\beta(\alpha i+1)+j-1 \\
k
\end{array}\right)\left(\begin{array}{l}
n \\
i
\end{array}\right)\left(\begin{array}{c}
l \\
m
\end{array}\right) .
$$

Also, we can write the PDF of GWE-G in the form:

$$
f(x, \alpha, \beta, \lambda, \sigma, n)=\frac{\alpha \beta \lambda}{B\left(\frac{1}{\alpha}, n+1\right)} \mathrm{e}^{\lambda x-\sigma\left(\mathrm{e}^{2 x}-1\right)} \sum_{i=0}^{n}(-1)^{i}\left(\begin{array}{l}
n \\
i
\end{array}\right) A^{\beta(\alpha i+1)-1} .
$$

Now, using the power series (12) in the last term of above equation:

$$
\begin{aligned}
& f(x, \alpha, \beta, \lambda, \sigma, n) \\
& =\frac{\alpha \beta \lambda \sigma}{B\left(\frac{1}{\alpha}, n+1\right)} \sum_{i=0}^{n} \sum_{j=0}^{\infty}(-1)^{i+j}\left(\begin{array}{c}
n \\
i
\end{array}\right)\left(\begin{array}{c}
\beta(\alpha i+1)-1 \\
j
\end{array}\right)\left[\mathrm{e}^{\lambda x-\sigma(j+1)\left(\mathrm{e}^{\lambda x}-1\right)}\right] \\
& =\sum_{i=0}^{n} \sum_{j=0}^{\infty} i_{i, j} g(x, \lambda, \sigma(j+1)) .
\end{aligned}
$$

where,

$$
\iota_{i, j}=\frac{\alpha \beta \lambda \sigma(-1)^{i+j}}{B\left(\frac{1}{\alpha}, n+1\right)}\left(\begin{array}{c}
n \\
i
\end{array}\right)\left(\begin{array}{c}
\beta(\alpha i+1)-1 \\
j
\end{array}\right) .
$$

and $g(x, \lambda, \sigma(j+1))$ denotes the density function of Gompertz distribution with parameters $\lambda$ and $\sigma(j+1)$. Therefore, the density function of GWE-G can be expressed as an infinite linear combination of Gompertz densities.

\section{Statistical Properties}

In this part, Let us view different moments of GWE-G $(\alpha, \beta, \lambda, \sigma, n)$ distribution. By using the moment we can study some of the most important characteristics and features of a distribution, such as moment generating function, the moments, and interesting reliability properties such as mean residual lifetime.

\subsection{Moment Generating Function}

If $\mathrm{X}$ has the GWE-G $(x, \alpha, \beta, \lambda, \sigma, n)$ distribution, then the MGF is:

$$
M_{x}(t)=\sum_{i=0}^{n} \sum_{j=0 k, l=0}^{1} \sum_{m=0}^{\infty} \frac{\phi_{i, j, k, l, m}}{-t-\lambda(l-m+1)} ; \quad t+\lambda l+\lambda>\lambda m
$$

where, $\phi_{i, j, k, l, m}$ defined by Equation (18).

\subsection{Moments}

The GWE-G random variable has the $r^{\text {th }}$ moments about the origin are:

$$
\mu_{r}^{\prime}=\sum_{i=0}^{n} \sum_{j=0}^{1} \sum_{k, l=0}^{\infty} \sum_{m=0}^{l} \frac{(-1)^{r+1} \Gamma(r+1)}{[\lambda(l-m+1)]^{r+1}} \phi_{i, j, k, l}
$$




\section{Mean}

For $r=1$ Equation (21), yields the mean of GWE-G that is given by:

$$
\mu=\sum_{i=0}^{n} \sum_{j=0 k, l,=0}^{1} \sum_{m=0}^{\infty} \frac{1}{[\lambda(l-m+1)]^{2}} \phi_{i, j, k, l}
$$

\subsection{Central Moments and Cumulants}

The central moments $\left(\mu_{r}\right)$ and cumulants $\left(\kappa_{r}\right)$ of X can be calculated as

$$
\begin{aligned}
\mu_{r} & =\sum_{s=0}^{r}(-1)^{r-s}\left(\begin{array}{l}
r \\
s
\end{array}\right)\left(\mu_{1}^{\prime}\right)^{r-s} \mu_{s}^{\prime} \\
& =\sum_{i=0}^{n} \sum_{j=0 k, l=0}^{1} \sum_{m=0}^{\infty} \sum_{m=0}^{l}\left(\begin{array}{l}
r \\
s
\end{array}\right) \frac{(-1)^{r+1}(\mu)^{r-s} \phi_{i, j, k, l, m} \Gamma(s+1)}{[\lambda(l-m+1)]^{s+1}}
\end{aligned}
$$

and

$$
\kappa_{r}=\mu_{r}^{\prime}-\sum_{s=1}^{r-1}\left(\begin{array}{l}
r-1 \\
s-1
\end{array}\right) \kappa_{s} \mu_{r-s}^{\prime}
$$

respectively, where $\mu_{1}=\mu_{1}^{\prime}$. Then, $\mu_{2}=\mu_{2}^{\prime}-\mu_{1}^{\prime 2}, \mu_{3}=\mu_{3}^{\prime}-3 \mu_{2}^{\prime} \mu_{1}^{\prime}+2 \mu_{1}^{\prime 3}$, $\mu_{4}=\mu_{4}^{\prime}-4 \mu_{3}^{\prime} \mu_{1}^{\prime}+6 \mu_{2}^{\prime} \mu_{1}^{\prime 2}-3 \mu_{1}^{\prime 4}$, etc. Also, the skewness $\gamma_{1}=\mu_{3} / \mu_{2}^{3 / 2}$ and kurtosis $\gamma_{2}=\mu_{4} / \mu_{2}^{2}$ follow from the second, third and fourth moments.

Variance

For $r=2$ Equation (23), the variance of GWE-G distribution is given by:

$$
\mu_{2}=\sum_{i=0}^{n} \sum_{j=0}^{1} \sum_{k, l=0}^{\infty} \sum_{m=0}^{l} \sum_{s=0}^{2}\left(\begin{array}{l}
2 \\
s
\end{array}\right) \frac{(-1)^{3}(\mu)^{2-s} \phi_{i, j, k, l, m} \Gamma(s+1)}{[\lambda(l-m+1)]^{s+1}}
$$

\subsection{The Mean Deviation}

Let $\mathrm{X}$ be a random variable that follows GWE-G distribution with median $\mathrm{m}$ and mean $\mu$. In this subsection, we inferred the mean deviation from the mean and the median.

\subsubsection{The Mean Deviation from the Mean Can Be Found from the following Theorem}

Theorem 1. The form of the mean deviation from the mean of the GWE-G distribution is

$$
\begin{aligned}
E(|X-\mu|)= & \sum_{i=0}^{n} \sum_{j, k=0}^{\infty} \sum_{l=0}^{k} \frac{2 \alpha(-1)^{i+j+k+l}}{(\alpha i+1) B\left(\frac{1}{\alpha}, n+1\right)}\left(\begin{array}{c}
n \\
i
\end{array}\right)\left(\begin{array}{c}
k \\
l
\end{array}\right) \\
& \times\left(\begin{array}{c}
\beta(\alpha i+1)-1 \\
j
\end{array}\right) \frac{(\sigma j)^{k}}{k ![\lambda(k-l)]}\left[\mathrm{e}^{\lambda \mu(k-l)}-1\right]
\end{aligned}
$$

Proof: The mean deviation from the mean can be defined as

$$
\begin{aligned}
E(|X-\mu|) & =\int_{0}^{\infty}|x-\mu| f(x) \mathrm{d} x \\
& =2 \int_{0}^{\mu}(\mu-x) f(x) \mathrm{d} x
\end{aligned}
$$




$$
\begin{aligned}
& =2 \mu F(\mu)-2 \int_{0}^{\mu} x f(x) \mathrm{d} x \\
& =2 \int_{0}^{\mu} F(x) \mathrm{d} x
\end{aligned}
$$

where, $\mathrm{d}[t G(t)]=G(t) \mathrm{d} t+t \mathrm{~d} G(t)$. Using Equation (15) we obtain

$$
\begin{aligned}
E(|x-\mu|)= & \sum_{i=0}^{n} \sum_{j, k=0 l=0}^{\infty} \sum^{k} \frac{2 \alpha(-1)^{i+j+k+l}}{(\alpha i+1) B\left(\frac{1}{\alpha}, n+1\right)}\left(\begin{array}{l}
n \\
i
\end{array}\right)\left(\begin{array}{l}
k \\
l
\end{array}\right) \\
& \times\left(\begin{array}{c}
\beta(\alpha i+1)-1) \frac{(\sigma j)^{k}}{k !} \int_{0}^{\mu} \mathrm{e}^{\lambda x(k-l)} \mathrm{d} x \\
j
\end{array}\right) \\
& \sum_{i=0}^{n} \sum_{j, k=0 l=0}^{\infty} \sum_{l=0}^{k} \frac{2 \alpha(-1)^{i+j+k+l}}{(\alpha i+1) B\left(\frac{1}{\alpha}, n+1\right)}\left(\begin{array}{l}
n \\
i
\end{array}\right)\left(\begin{array}{l}
k \\
l
\end{array}\right) \\
& \times\left(\begin{array}{c}
\beta(\alpha i+1)-1 \\
j
\end{array}\right) \frac{(\sigma j)^{k}}{k ![\lambda(k-l)]}\left[\mathrm{e}^{\lambda \mu(k-l)-1}\right]
\end{aligned}
$$

Hence, the theorem is proved.

\subsubsection{The Mean Deviation from the Median Can Be Found from the Following Theorem}

Theorem 2. The mean deviation from the median $(m)$ of the GWE-G distribution is in the form

$$
\begin{aligned}
E(|X-m|)= & \mu-m+2 \sum_{i=0}^{n} \sum_{j, k=0}^{\infty} \sum_{l=0}^{k} \frac{\alpha(-1)^{i+j+k+l}}{(\alpha i+1) B\left(\frac{1}{\alpha}, n+1\right)}\left(\begin{array}{l}
n \\
i
\end{array}\right)\left(\begin{array}{l}
k \\
l
\end{array}\right) \\
& \times\left(\begin{array}{c}
\beta(\alpha i+1)-1 \\
j
\end{array}\right) \frac{(\sigma j)^{k}}{k ![\lambda(k-l)]}\left[\mathrm{e}^{\lambda m(k-l)}-1\right]
\end{aligned}
$$

Proof: The mean deviation from the median can be defined as

$$
\begin{aligned}
E(|X-m|) & =\int_{0}^{\infty}|x-m| f(x) \mathrm{d} x \\
& =2 \int_{0}^{m}(m-x) f(x) \mathrm{d} x+\int_{0}^{\infty}(x-m) f(x) \mathrm{d} x \\
& =2 m F(m)+\mu-m-2 \int_{0}^{m} x f(x) \mathrm{d} x \\
& =\mu-m+2 \int_{0}^{m} F(x) \mathrm{d} x
\end{aligned}
$$

Using Equation (15) we obtain

$$
\begin{aligned}
E(|X-m|)= & \mu-m+2 \sum_{i=0}^{n} \sum_{j, k=0}^{\infty} \sum_{l=0}^{k} \frac{\alpha(-1)^{i+j+k+l}}{(\alpha i+1) B\left(\frac{1}{\alpha}, n+1\right)}\left(\begin{array}{l}
n \\
i
\end{array}\right)\left(\begin{array}{c}
k \\
l
\end{array}\right) \\
& \times\left(\begin{array}{c}
\beta(\alpha i+1)-1 \\
j
\end{array}\right) \frac{(\sigma j)^{k}}{k !} \int_{0}^{m} \mathrm{e}^{\lambda x(k-l)} \mathrm{d} x \\
= & \mu-m+2 \sum_{i=0}^{n} \sum_{j, k=0 l=0}^{\infty} \sum^{k} \frac{\alpha(-1)^{i+j+k+l}}{(\alpha i+1) B\left(\frac{1}{\alpha}, n+1\right)}\left(\begin{array}{l}
n \\
i
\end{array}\right)\left(\begin{array}{c}
k \\
l
\end{array}\right) \\
& \times\left(\begin{array}{c}
\beta(\alpha i+1)-1 \\
j
\end{array}\right) \frac{(\sigma j)^{k}}{k ![\lambda(k-l)]}\left[\mathrm{e}^{\lambda m(k-l)}-1\right]
\end{aligned}
$$


Hence, the theorem is proved.

\subsection{The Mode}

The mode for the GWE-G distribution can be found by differentiating $f(x)$ with respect to $x$, thus, from Equation (8)

$$
\begin{aligned}
f^{\prime}(x)= & f(x)\left[\lambda-\lambda \sigma \mathrm{e}^{\lambda x}+\frac{\lambda \sigma(\beta-1) \mathrm{e}^{\lambda x}[1-A(x)]}{A(x)}\right. \\
& \left.-\frac{n \alpha \beta \lambda \sigma \mathrm{e}^{\lambda x}[1-A(x)] A^{\alpha \beta-1}(x)}{1-A^{\alpha \beta}(x)}\right]
\end{aligned}
$$

By equating Equation (27) with zero, we get

$$
1-\sigma \mathrm{e}^{\lambda x}+\frac{\sigma(\beta-1) \mathrm{e}^{\lambda x}[1-A(x)]}{A(x)}-\frac{n \alpha \beta \sigma \mathrm{e}^{\lambda x}[1-A(x)] A^{\alpha \beta-1}(x)}{1-A^{\alpha \beta}(x)}=0
$$

Then, the mode of GWE-G distribution can be found numerically by solving Equation (28).

\section{Rényi Entropy}

Entropy is a measurement representation of the degree of disorder or uncertainty in a system. The Rényi entropy is defined by

$$
I_{R}(\gamma)=(1-\gamma)^{-1} \log \int_{R} f^{\gamma}(x) \mathrm{d} x, \quad(\gamma>0, \gamma \neq 1)
$$

In this case

$$
\begin{aligned}
f^{\gamma}(x) & =\left[\frac{\alpha \beta \lambda \sigma}{B\left(\frac{1}{\alpha}, n+1\right)}\right]^{\gamma} \mathrm{e}^{\lambda \gamma x} A^{(\beta-1) \gamma}(x)[1-A(x)]^{\gamma}\left[1-A^{\alpha \beta}(x)\right]^{n \gamma} \\
& =\left[\frac{\alpha \beta \lambda \sigma}{B\left(\frac{1}{\alpha}, n+1\right)}\right]^{\gamma} \sum_{i=0}^{n \gamma}(-1)^{i}\left(\begin{array}{c}
n \gamma \\
i
\end{array}\right) \mathrm{e}^{\lambda \gamma \gamma} A^{\beta(\alpha i+\gamma)-\gamma}(x)[1-A(x)]^{\gamma} \\
& =\left[\frac{\alpha \beta \lambda \sigma}{B\left(\frac{1}{\alpha}, n+1\right)}\right]^{\gamma} \sum_{i=0}^{n \gamma} \sum_{j=0}^{\gamma}(-1)^{i+j}\left(\begin{array}{c}
n \gamma \\
i
\end{array}\right)\left(\begin{array}{c}
\gamma \\
j
\end{array}\right) \mathrm{e}^{\lambda \gamma x} A^{\beta(\alpha i+\gamma)-\gamma+j}(x)
\end{aligned}
$$

We expand the term $A^{\beta(\alpha i+\gamma)-\gamma+j}(x)=\left[1-\exp ^{-\sigma\left(\mathrm{e}^{\lambda x}-1\right)}\right]^{\beta(\alpha i+\gamma)-\gamma+j}$, the $f^{\gamma}(x)$ can be rewritten as:

$$
f^{\gamma}(x)=\left[\frac{\alpha \beta \lambda \sigma}{B\left(\frac{1}{\alpha}, n+1\right)}\right]^{\gamma} \sum_{i=0}^{n \gamma} \sum_{j=0}^{\gamma} \sum_{k=0}^{\infty}(-1)^{i+j+k}\left(\begin{array}{c}
n \gamma \\
i
\end{array}\right)\left(\begin{array}{c}
\gamma \\
j
\end{array}\right) \times\left(\begin{array}{c}
\beta(\alpha i+\gamma)-\gamma+j \\
k
\end{array}\right) \exp \left[\lambda \gamma x-k \sigma\left(\mathrm{e}^{\lambda x}-1\right)\right]
$$

Using power series expansion for $\left(\exp \left[\lambda \gamma x-\sigma k\left(\mathrm{e}^{\lambda x}-1\right)\right]\right)$, we have: 


$$
\begin{aligned}
& f^{\gamma}(x)=\left[\frac{\alpha \beta \lambda \sigma}{B\left(\frac{1}{\alpha}, n+1\right)}\right]^{\gamma} \sum_{i=0}^{n \gamma} \sum_{j=0 k, l=0}^{\gamma} \sum^{\infty}(-1)^{i+j+k+l}\left(\begin{array}{c}
n \gamma \\
i
\end{array}\right)\left(\begin{array}{c}
\gamma \\
j
\end{array}\right) \\
& \times\left(\begin{array}{c}
\beta(\alpha i+\gamma)-\gamma+j \\
k
\end{array}\right) \frac{\left[\lambda \gamma x-k \sigma\left(\mathrm{e}^{\lambda x}-1\right)\right]^{l}}{l !} \\
& \text { when we expand the term }\left[\lambda \gamma x-k \sigma\left(\mathrm{e}^{\lambda x}-1\right)\right]^{l} \\
& f^{\gamma}(x)=\left[\frac{\alpha \beta \lambda \sigma}{B\left(\frac{1}{\alpha}, n+1\right)}\right]^{\gamma} \sum_{i=0}^{n \gamma} \sum_{j=0}^{\gamma} \sum_{k, l=0}^{\infty} \sum_{m=0}^{l}(-1)^{i+j+k+l+m}\left(\begin{array}{c}
n \gamma \\
i
\end{array}\right)\left(\begin{array}{c}
\gamma \\
j
\end{array}\right) \\
& \times\left(\begin{array}{c}
\beta(\alpha i+\gamma)-\gamma+j \\
k
\end{array}\right) \frac{(\sigma k)^{m}(\lambda \gamma)^{l-m}}{l !} x^{l-m}\left(\mathrm{e}^{\lambda x}-1\right)^{m} \\
& f^{\gamma}(x)=\left[\frac{\alpha \beta \lambda \sigma}{B\left(\frac{1}{\alpha}, n+1\right)}\right]^{\gamma} \sum_{i=0}^{n \gamma} \sum_{j=0 k, l=0}^{\gamma} \sum_{m=0 s, q=0}^{l} \sum^{\infty}(-1)^{i+j+k+l+m=s+q}\left(\begin{array}{c}
n \gamma \\
i
\end{array}\right)\left(\begin{array}{c}
\gamma \\
j
\end{array}\right) \\
& \times\left(\begin{array}{c}
\beta(\alpha i+\gamma)-\gamma+j \\
k
\end{array}\right)\left(\begin{array}{c}
m \\
s
\end{array}\right) \frac{(\sigma k)^{m}(\lambda)^{l-m+q}(\gamma)^{l-m}(m-s)^{q}}{l ! q !} x^{l-m+q}
\end{aligned}
$$

Hence, the Rényi entropy is given by

$$
I_{R}(\gamma)=(1-\gamma)^{-1} \log \left[\sum_{i=0}^{n \gamma} \sum_{j=0}^{\gamma} \sum_{k, l=0}^{\infty} \sum_{m=0}^{l} \sum_{s, q=0}^{\infty} \varpi_{i, j, k, l, m, s, q} \frac{x^{l+q-m+1}}{l+q-m+1}\right]
$$

when

$$
\varpi_{i, j, k, l, m, s, q}=\frac{(-1)^{i+j+k+l+m=s+q}\left(\begin{array}{c}
n \gamma \\
i
\end{array}\right)\left(\begin{array}{c}
\gamma \\
j
\end{array}\right)\left(\begin{array}{c}
\beta(\alpha i+\gamma)-\gamma+j \\
k
\end{array}\right)\left(\begin{array}{c}
m \\
s
\end{array}\right)(\alpha \beta)^{\gamma}(\sigma)^{m+\gamma}(k)^{m}(\lambda)^{l-m+q+\gamma}(\gamma)^{l-m}(m-s)^{q}}{l ! q !\left(B\left(\frac{1}{\alpha}, n+1\right)\right)^{\gamma}}
$$

\section{Reliability Measures of GWE-G}

Here, we derive the expression for the mean and strong mean inactivity time functions, mean of residual lifetime of the GWE-G model.

\subsection{Mean Residual Time}

One of the well known properties of the lifetime distribution is mean residual lifetime (MRL). For the GWE-G distribution, it can be written as

$$
m(t)=\frac{1}{S(t)} \int_{t}^{\infty} S(x) \mathrm{d} x
$$

by using Equation (17) the MRL can be written as: 


$$
m(t)=\frac{\sum_{i=0}^{n} \sum_{j=0}^{1} \sum_{k, l=0}^{\infty} \sum_{m=0}^{l} v_{i, j, k, l} \frac{\mathrm{e}^{\lambda x(l-m)}}{\lambda(m-l)}}{\sum_{i=0}^{n} \sum_{j=0}^{1} \sum_{k, l=0}^{\infty} \sum_{m=0}^{l} v_{i, j, k, l, m}\left(\mathrm{e}^{\lambda x(l-m)}\right)} ; \quad t>0 .
$$

\subsection{Mean Inactivity and Strong Mean Inactivity Time}

The mean inactivity time (MIT) and strong mean inactivity time (SMIT) functions are significant to describe the time which had elapsed since the failure in many applications. Many properties and applications of MIT and SMIT functions can be found in Kayid and Ahmad [19], and Kayid and Izadkhah [20]. Let $\mathrm{X}$ be a lifetime random variable with CDF, $F(\cdot)$. Then the (MIT) and (SMIT) are defined as, respectively:

$$
\xi_{M I T}(t)=\frac{1}{F(t)} \int_{0}^{t} F(x) \mathrm{d} x
$$

and

$$
\vartheta_{M I T}(t)=\frac{1}{F(t)} \int_{0}^{t} 2 x F(x) \mathrm{d} x
$$

by using the expansion form of CDF that given in Equation (16) and after some simple calculations, we obtain the MIT of GWE-G distribution as:

$$
\xi_{M I T}=\frac{\sum_{i=0}^{n} \sum_{m, k, j=0}^{\infty} \sum_{l=0}^{k} \rho_{i, j, k, l, m} \frac{t^{m+1}}{m+1}}{\sum_{i=0}^{n} \sum_{m, k, j=0}^{\infty} \sum_{l=0}^{k} \rho_{i, j, k, l, m} t^{m}} ; \quad t>0 .
$$

Similarly, we obtain the SMIT of the GWE-G distribution as:

$$
\vartheta_{\text {SMIT }}=\frac{\sum_{i=0}^{n} \sum_{m, k, j=0}^{\infty} \sum_{l=0}^{k} \rho_{i, j, k, l, m} \frac{t^{m+2}}{m+2}}{\sum_{i=0}^{n} \sum_{m, k, j=0}^{\infty} \sum_{l=0}^{k} \rho_{i, j, k, l, m} t^{m}} ; \quad t>0 .
$$

We observed from Table 1 and Table 2 that the MIT and SMIT are decreasing for increasing values of $\alpha$ and $\beta$. Also from Table 3 the MIT and SMIT are increasing for increasing values of $n$.

\section{Estimation and Inference}

In this section, the ML method is considered to estimate the parameters of GWE-G $(x, \alpha, \beta, \lambda, \sigma, n)$ distribution. Let $\left(X_{1}, X_{2}, \cdots, X_{m}\right)$ be a random sample with size $m$ from the GWE-G with PDF and CDF given, respectively, by Equation (8) and Equation (9). Also, we assume that $\Theta=(\alpha, \beta, \lambda, \sigma)^{\mathrm{T}}$ is the $(r * l)$ unknown parameter vectors. Based on the Equation (8), the log-likelihood function is defined by:

$$
l(\Theta)=l\left(x_{1}, x_{2}, \cdots, x_{n} \mid \Theta\right)=\log L_{m}(x)
$$

where,

$$
L_{m}(x)=\prod_{i=1}^{m} f\left(x_{i}, \Theta\right)
$$


Table 1. MIT and SMIT of GWE-G distribution at $t=8$ and $n=2$.

\begin{tabular}{|c|c|c|c|}
\hline$\alpha$ & $\beta=2.5, \lambda=0.4, \sigma=1.5$ & MIT & SMIT \\
\hline 0.01 & & 7.29476 & 63.3085 \\
\hline 0.8 & & 6.9773 & 62.716 \\
\hline 0.5 & & 6.72204 & 62.0715 \\
\hline 5 & & 6.57676 & 61.6221 \\
\hline 7 & & 6.517 & 61.4162 \\
\hline
\end{tabular}

Table 2. MIT and SMIT of GWE-G distribution at $t=8$ and $n=3$.

\begin{tabular}{|c|c|c|c|}
\hline$\beta$ & $\alpha=3.5, \lambda=0.4, \sigma=2.5$ & MIT & SMIT \\
\hline 0.05 & & 7.99905 & 63.9999 \\
\hline 0.7 & & 7.72049 & 63.8527 \\
\hline 2 & & 7.26157 & 63.3189 \\
\hline 3.8 & & 6.92267 & 62.696 \\
\hline 5 & & 6.7765 & 62.3633 \\
\hline
\end{tabular}

Table 3. MIT and SMIT of GWE-G distribution at $t=8$.

\begin{tabular}{|c|c|c|c|}
\hline$n$ & $\alpha=6, \beta=0.3, \lambda=0.5, \sigma=1$ & MIT & SMIT \\
\hline 1 & & 7.66701 & 63.6709 \\
\hline 2 & & 7.74102 & 63.7975 \\
\hline 3 & & 7.78211 & 63.8555 \\
\hline 4 & & 7.80898 & 63.8883 \\
\hline 5 & & 7.82824 & 63.9094 \\
\hline
\end{tabular}

is the likelihood function. We can derive the likelihood function of GWE-G distribution as:

$$
L_{m}(x)=\frac{(\alpha \beta \sigma \lambda)^{m}}{B\left[\frac{1}{\alpha}, n+1\right]^{m}} \prod_{i=1}^{m}\left[1-A\left(x_{i}\right)\right]\left[A^{\beta-1}\left(x_{i}\right)\right]\left[1-A^{\beta \alpha}\left(x_{i}\right)\right]^{n} \mathrm{e}^{\lambda x_{i}}
$$

thus, the log-likelihood function of GWE-G distribution is obtained as:

$$
\begin{aligned}
\ell(\Theta)= & m \log \alpha+m \log \beta+m \log \lambda+m \log \sigma+m \sigma \\
& -\sigma \sum_{i=1}^{m} \exp ^{\lambda x_{i}}+(\beta-1) \sum_{i=1}^{m} \log A\left(x_{i}\right)+\lambda \sum_{i=1}^{m} x_{i} \\
& +n \sum_{i=1}^{m} \log \left[1-A^{\alpha \beta}\left(x_{i}\right)\right]-m \log \left(B\left[\frac{1}{\alpha}, n+1\right]\right)
\end{aligned}
$$

The first derivatives of Equation (33) with respect to $\alpha, \beta, \lambda$ and $\sigma$ respectively is given by:

$$
\frac{\partial \ell(\Theta)}{\partial \alpha}=\frac{m}{\alpha}-n \beta \sum_{i=0}^{m} \frac{A^{\alpha \beta}\left(x_{i}\right) \log A\left(x_{i}\right)}{1-A^{\alpha \beta}\left(x_{i}\right)}+\frac{m}{\alpha^{2}}\left[\psi^{(0)}\left(\frac{1}{\alpha}\right)-\psi^{(0)}\left(\frac{1}{\alpha}+n+1\right)\right]
$$




$$
\begin{gathered}
\frac{\partial \ell(\Theta)}{\partial \beta}=\frac{m}{\beta}+\sum_{i=0}^{m} \log A\left(x_{i}\right)-n \alpha \sum_{i=0}^{m} \frac{A^{\alpha \beta}\left(x_{i}\right) \log A\left(x_{i}\right)}{1-A^{\alpha \beta}\left(x_{i}\right)} \\
\frac{\partial \ell(\Theta)}{\partial \sigma}=m+\frac{m}{\sigma}-\sum_{i=0}^{m} \mathrm{e}^{\lambda\left(x_{i}\right)}+(\beta-1) \sum_{i=0}^{m} \frac{\left(\mathrm{e}^{\lambda\left(x_{i}\right)}-1\right)\left[1-A\left(x_{i}\right)\right]}{A\left(x_{i}\right)} \\
-n \alpha \beta \sum_{i=0}^{m} \frac{\left(\mathrm{e}^{\lambda\left(x_{i}\right)}-1\right)\left[1-A\left(x_{i}\right)\right] A^{\alpha \beta}\left(x_{i}\right)}{1-A^{\alpha \beta}\left(x_{i}\right)} \\
\frac{\partial \ell(\Theta)}{\partial \lambda}=\frac{m}{\lambda}-\sigma \sum_{i=0}^{m} x_{i} \mathrm{e}^{\lambda\left(x_{i}\right)}+(\beta-1) \sum_{i=0}^{m} \frac{\sigma x_{i} \exp \left[\lambda x_{i}-\sigma\left(\mathrm{e}^{\lambda\left(x_{i}\right)}-1\right)\right]}{A\left(x_{i}\right)} \\
+\sum_{i=0}^{m} x_{i}-n \alpha \beta \sigma \sum_{i=0}^{m} \frac{x_{i} A^{\alpha \beta-1}\left(x_{i}\right) \exp \left[\lambda x_{i}-\sigma\left(\mathrm{e}^{\lambda\left(x_{i}\right)}-1\right)\right]}{1-A^{\alpha \beta}\left(x_{i}\right)}
\end{gathered}
$$

where,

$$
\psi^{(m)}(\alpha)=\frac{\mathrm{d}^{m+1}}{\mathrm{~d} \alpha^{m+1}} \log \Gamma(\alpha)=\frac{\mathrm{d}^{m} \Gamma^{\prime}(\alpha)}{\mathrm{d} a^{m} \Gamma(\alpha)}=\frac{\mathrm{d}^{m}}{\mathrm{~d} \alpha^{m}} \psi^{(0)}(\alpha)
$$

is the polygamma function of order $m$ and it is defined by $(m+1)^{\text {th }}$ derivative of the logarithm of the gamma function, as $\alpha$ particular case, $\psi^{(0)}(\alpha)=\frac{\Gamma^{\prime}(\alpha)}{\Gamma(\alpha)}$ is called digamma function.

Equate the Equations (34)-(37) to zero and solving them simultaneously yield the maximum likelihood estimators (MLEs) of GWE-G distribution parameters. Clearly, these equations are not in explicit form, the solutions can be found by using a numerical method such as the Newton-Raphson procedure to obtain the MLEs of the parameters $\alpha, \beta, \lambda$ and $\sigma$.

To obtain the asymptotic confidence intervals (CIs) for the parameters of the GWE-G distribution, the $4 \times 4 \quad I_{m}(\Theta)=I_{m}(\alpha, \beta, \lambda, \sigma)$ is required. Under certain regularity conditions, the MLEs asymptotically have (for large samples) a multivariate normal distribution with mean vector $\underline{0}=(0,0,0,0)^{\mathrm{T}}$ and variance-covariance matrix, which is given by the inverse of Fisher information matrix: $I_{m}^{-1}(\hat{\Theta})=I_{m}^{-1}(\hat{\alpha}, \hat{\beta}, \hat{\lambda}, \hat{\sigma})$ for more details about asymptotic confidence intervals. The $I_{m}(\Theta)$ depends on $\Theta$, the observed Fisher information matrix $I_{m}(\hat{\Theta})$ may be used instead of the $I_{m}(\Theta)$ in the estimation of the variance of MLEs. The $4 \times 4$ observed information matrix, $I_{m}(\hat{\Theta})=I_{m}(\hat{\alpha}, \hat{\beta}, \hat{\lambda}, \hat{\sigma})$ is:

$$
I_{m}(\hat{\Theta})=-\left(\begin{array}{llll}
I_{\alpha \alpha} & I_{\alpha \beta} & I_{\alpha \lambda} & I_{\alpha \sigma} \\
I_{\beta \alpha} & I_{\beta \beta} & I_{\beta \lambda} & I_{\beta \sigma} \\
I_{\lambda \alpha} & I_{\lambda \beta} & I_{\lambda \lambda} & I_{\lambda \sigma} \\
I_{\sigma \alpha} & I_{\sigma \beta} & I_{\sigma \lambda} & I_{\sigma \sigma}
\end{array}\right)_{\alpha=\hat{\alpha}, \beta=\hat{\beta}, \lambda=\hat{\lambda}, \sigma=\hat{\sigma}} .
$$

where, $I_{i j}(\underline{\theta})=E\left(\frac{\partial^{2} \log \ell}{\partial \theta_{i} \partial \theta_{j}}\right)$ Regrettably, the accurate mathematical expressions for the above expectation are very hard to obtain. Therefore, the observed 
Fisher information matrix is given by $I_{i j}=\frac{\partial^{2} \log \ell}{\partial \theta_{i} \partial \theta_{j}}$ which is obtained by dropping the expectation on operation $\mathrm{E}$.

The elements of the $I_{m}(\hat{\Theta})$ are given by the following equations:

$$
\begin{aligned}
& I_{\alpha \alpha}=\frac{-m}{\alpha^{2}}-\frac{m}{\alpha^{3}}\left[\frac{\psi^{1}\left(\frac{1}{\alpha}\right)}{\alpha}-\frac{\psi^{1}\left(\frac{1}{\alpha}+n+1\right)}{\alpha}\right] \\
& +2\left[\psi^{0}\left(\frac{1}{\alpha}\right)-\psi^{0}\left(\frac{1}{\alpha}+n+1\right)\right]-n \beta^{2} \sum_{i=1}^{m} \frac{A^{\alpha \beta}\left(x_{i}\right)\left(\log A\left(x_{i}\right)\right)^{2}}{\left(1-A^{\alpha \beta}\left(x_{i}\right)\right)^{2}} \\
& I_{\beta \beta}=\frac{-m}{\beta^{2}}-n \alpha^{2} \sum_{i=1}^{m} \frac{A^{\alpha \beta}\left(x_{i}\right)\left(\log A\left(x_{i}\right)\right)^{2}}{\left(1-A^{\alpha \beta}\left(x_{i}\right)\right)^{2}} \\
& I_{\alpha \beta}=I_{\beta \alpha}=-n\left[\sum_{i=1}^{m} \frac{\alpha \beta A^{\alpha \beta}\left(x_{i}\right)\left(\log A\left(x_{i}\right)\right)^{2}}{\left(1-A^{\alpha \beta}\left(x_{i}\right)\right)^{2}}+\frac{A^{\alpha \beta}\left(x_{i}\right) \log A\left(x_{i}\right)}{1-A^{\alpha \beta}\left(x_{i}\right)}\right] \\
& I_{\sigma \alpha}=I_{\alpha \sigma}=n \beta \sum_{i=1}^{m} \frac{\left(1-\mathrm{e}^{\lambda x_{i}}\right) A^{\alpha \beta-1}\left(x_{i}\right) \exp \left[\left(1-\mathrm{e}^{\lambda x}\right) \sigma\right]\left[1-A^{\alpha \beta}\left(x_{i}\right)+\alpha \beta \log A\left(x_{i}\right)\right]}{\left(1-A^{\alpha \beta}\left(x_{i}\right)\right)^{2}} \\
& I_{\alpha \lambda}=I_{\lambda \alpha}=-n \beta \sum_{i=1}^{m} \frac{\sigma x_{i} A^{\alpha \beta-1}\left(x_{i}\right) \exp \left[\left(1-\mathrm{e}^{\lambda x_{i}}\right) \sigma+\lambda x_{i}\right]\left[1-A^{\alpha \beta}\left(x_{i}\right)+\alpha \beta \log A\left(x_{i}\right)\right]}{\left(1-A^{\alpha \beta}\left(x_{i}\right)\right)^{2}} \\
& I_{\beta \lambda}=I_{\lambda \beta} \\
& =\sum_{i=1}^{m} \frac{\sigma x_{i} \exp \left[\left(1-\mathrm{e}^{\lambda x_{i}}\right) \sigma+\lambda x_{i}\right]}{A\left(x_{i}\right)} \\
& -n \alpha \sum_{i=1}^{m} \frac{\sigma x_{i} A^{\alpha \beta-1}\left(x_{i}\right) \exp \left[\left(1-\mathrm{e}^{\lambda x_{i}}\right) \sigma+\lambda x_{i}\right]\left[1-A^{\alpha \beta}\left(x_{i}\right)+\alpha \beta \log A\left(x_{i}\right)\right]}{\left(1-A^{\alpha \beta}\left(x_{i}\right)\right)^{2}} \\
& I_{\beta \sigma}=I_{\sigma \beta} \\
& =-\sum_{i=1}^{m} \frac{\left(1-\mathrm{e}^{\lambda x_{i}}\right) \exp \left[\left(1-\mathrm{e}^{\lambda x_{i}}\right) \sigma\right]}{A\left(x_{i}\right)} \\
& +n \alpha \sum_{i=1}^{m} \frac{\left(1-\mathrm{e}^{\lambda x_{i}}\right) A^{\alpha \beta-1}\left(x_{i}\right) \exp \left[\left(1-\mathrm{e}^{\lambda x_{i}}\right) \sigma\right]\left[1-A^{\alpha \beta}\left(x_{i}\right)+\alpha \beta \log A\left(x_{i}\right)\right]}{\left(1-A^{\alpha \beta}\left(x_{i}\right)\right)^{2}} \\
& I_{\lambda \lambda}=-\frac{m}{\lambda^{2}}-\sigma \sum_{i=1}^{m} x_{i}^{2} \mathrm{e}^{\lambda x_{i}}-n \alpha \beta \sigma \sum_{i=1}^{m}\left[\frac{x_{i}^{2}\left(1-\sigma \mathrm{e}^{\lambda x_{i}}\right) \exp \left[\left(1-\mathrm{e}^{\lambda x_{i}}\right) \sigma+\lambda x_{i}\right] A^{\alpha \beta-1}\left(x_{i}\right)}{1-A^{\alpha \beta}\left(x_{i}\right)}\right. \\
& +\frac{\sigma(\alpha \beta-1) x_{i}^{2} \exp \left[2\left(1-\mathrm{e}^{\lambda x_{i}}\right) \sigma+2 \lambda x_{i}\right] A^{\alpha \beta-2}\left(x_{i}\right)}{1-A^{\alpha \beta}\left(x_{i}\right)} \\
& \left.+\frac{\alpha \beta \sigma x_{i}^{2} \exp \left[2\left(1-\mathrm{e}^{\lambda x_{i}}\right) \sigma+2 \lambda x_{i}\right] A^{2 \alpha \beta-2}\left(x_{i}\right)}{\left(1-A^{\alpha \beta}\left(x_{i}\right)\right)^{2}}\right]
\end{aligned}
$$




$$
\left.\begin{array}{c}
+(\beta-1) \sum_{i=1}^{m}\left[\frac{\sigma x_{i}^{2}\left(1-\sigma \mathrm{e}^{\lambda x_{i}}\right) \exp \left[\left(1-\mathrm{e}^{\lambda x_{i}}\right) \sigma+\lambda x_{i}\right]}{A\left(x_{i}\right)}\right] \\
\left.-\frac{\sigma^{2} x_{i}^{2} \exp \left[2\left(1-\mathrm{e}^{\lambda x_{i}}\right) \sigma+2 \lambda x_{i}\right]}{A^{2}\left(x_{i}\right)}\right] \\
\begin{array}{l}
I_{\sigma \lambda}=I_{\lambda \sigma} \\
=\sum_{i=1}^{m} x_{i} \mathrm{e}^{\lambda x_{i}}+(\beta-1) \sum_{i=1}^{m}\left[\frac{\sigma x_{i} \exp \left[\left(1-\mathrm{e}^{\lambda x_{i}}\right) \sigma+\lambda x_{i}\right]\left(1-\mathrm{e}^{\lambda x_{i}}\right)+x_{i} e^{\lambda x_{i}}-A\left(x_{i}\right) x_{i} e^{\lambda x_{i}}}{A\left(x_{i}\right)}\right. \\
\left.-\frac{\sigma x_{i} \exp \left[\left(1-\mathrm{e}^{\lambda x_{i}}\right) \sigma+\lambda x_{i}\right]\left(1-\mathrm{e}^{\lambda x_{i}}\right)\left[1-A\left(x_{i}\right)\right]}{A^{2}\left(x_{i}\right)}\right] \\
-n \alpha \beta \sum_{i=1}^{m}\left[\frac{x \mathrm{e}^{\lambda x_{i}} A^{\alpha \beta}\left(x_{i}\right)-x \mathrm{e}^{\lambda x_{i}} A^{\alpha \beta+1}\left(x_{i}\right)-\left(1-\mathrm{e}^{\lambda x_{i}}\right)\left[\alpha \beta A^{\alpha \beta-1}\left(x_{i}\right)-\alpha \beta A^{\alpha \beta}\left(x_{i}\right)-A^{\alpha \beta}\left(x_{i}\right)\right]}{1-A^{\alpha \beta}\left(x_{i}\right)}\right. \\
+\frac{\left.\alpha \beta\left(1-\mathrm{e}^{\lambda x_{i}}\right)\left[A^{2 \alpha \beta}\left(x_{i}\right)-A^{2 \alpha \beta-1}\left(x_{i}\right)\right]\right]}{\left(1-A^{\alpha \beta}\left(x_{i}\right)\right)^{2}} \\
I_{\sigma \sigma}=\frac{-m}{\sigma^{2}}-(\beta-1) \sum_{i=1}^{m} \frac{\left(\mathrm{e}^{\lambda x_{i}}-1\right)^{2} \exp \left[\left(1-\mathrm{e}^{\lambda x_{i}}\right) \sigma\right]}{A\left(x_{i}\right)^{2}} \\
-n \alpha \beta \sum_{i=1}^{m}\left(\mathrm{e}^{\lambda x_{i}}-1\right)^{2} \exp \left[\left(1-\mathrm{e}^{\lambda x_{i}}\right) \sigma\right] \\
\times\left[\frac{\alpha \beta A^{\alpha \beta-1}\left(x_{i}\right)-\alpha \beta A^{\alpha \beta}\left(x_{i}\right)-A^{\alpha \beta}\left(x_{i}\right)}{1-A^{\alpha \beta}\left(x_{i}\right)}\right.
\end{array} \\
\left(1-\alpha \beta A^{2 \alpha \beta}\left(x_{i}\right)\right. \\
\left.\left.+x_{i}\right)\right)^{2}
\end{array}\right]
$$

The approximate $(1-\delta) 100 \%$ CIs of the parameters of GWE-G $(\alpha, \beta, \lambda, \sigma)$ are respectively, given by: $\hat{\alpha} \pm Z_{\delta / 2} \sqrt{V(\hat{\alpha})}, \hat{\beta} \pm Z_{\delta / 2} \sqrt{V(\hat{\beta})}$ and $\hat{\lambda} \pm Z_{\delta / 2} \sqrt{V(\hat{\lambda})}, \quad \hat{\sigma} \pm Z_{\delta / 2} \sqrt{V(\hat{\sigma})}$ where, $V(\hat{\alpha}), V(\hat{\beta}), V(\hat{\lambda})$ and $V(\hat{\sigma})$ are the variances of $\hat{\alpha}, \hat{\beta}, \hat{\lambda}$ and $\hat{\sigma}$ which are given by the diagonal elements of $I_{m}^{-1}(\hat{\Theta})=I_{m}^{-1}(\hat{\alpha}, \hat{\beta}, \hat{\lambda}, \hat{\sigma})$ and $Z_{\delta / 2}$ is the upper $(\delta / 2)$ percentile of the standard normal distribution.

\section{Real Data Application}

In this section, we illustrate an application of the GWE-G distribution to the total milk production in the first birth of 107 cows from SINDI race. These cows are property of the original data is not in the interval $(0,1)$, and it was necessary to make Carnaúba farm which belongs to the Agropecuária Manoel Dantas Ltda (AMDA), located in Taperoá City, Paraíba (Brazil). a transformation given by $x_{i}=\left[y_{i}-\min \left(y_{i}\right)\right] /\left[\max \left(y_{i}\right)-\min \left(y_{i}\right)\right]$, for $i=1, \cdots, 107$. These data are presented in Table 4 and the values of $y_{i}$ are given in Table 3.1 of Brito ([21], p. 46). Also, Descriptive statistics of these data are tabulated in Table 5. 
Table 4. Proportion of total milk production.

\begin{tabular}{llllllllll}
\hline 0.4365 & 0.4260 & 0.5140 & 0.6907 & 0.7471 & 0.2605 & 0.6196 & 0.8781 & 0.4990 & 0.6058 \\
\hline 0.6891 & 0.5770 & 0.5394 & 0.1479 & 0.2356 & 0.6012 & 0.1525 & 0.5483 & 0.6927 & 0.7261 \\
0.3323 & 0.0671 & 0.2361 & 0.4800 & 0.5707 & 0.7131 & 0.5853 & 0.6768 & 0.5350 & 0.4151 \\
0.6789 & 0.4576 & 0.3259 & 0.2303 & 0.7687 & 0.4371 & 0.3383 & 0.6114 & 0.3480 & 0.4564 \\
0.7804 & 0.3406 & 0.4823 & 0.5912 & 0.5744 & 0.5481 & 0.1131 & 0.7290 & 0.0168 & 0.5529 \\
0.4530 & 0.3891 & 0.4752 & 0.3134 & 0.3175 & 0.1167 & 0.6750 & 0.5113 & 0.5447 & 0.4143 \\
0.5627 & 0.5150 & 0.0776 & 0.3945 & 0.4553 & 0.4470 & 0.5285 & 0.5232 & 0.6465 & 0.0650 \\
0.8492 & 0.8147 & 0.3627 & 0.3906 & 0.4438 & 0.4612 & 0.3188 & 0.2160 & 0.6707 & 0.6220 \\
0.5629 & 0.4675 & 0.6844 & 0.3413 & 0.4332 & 0.0854 & 0.3821 & 0.4694 & 0.3635 & 0.4111 \\
0.5349 & 0.3751 & 0.1546 & 0.4517 & 0.2681 & 0.4049 & 0.5553 & 0.5878 & 0.4741 & 0.3598 \\
0.7629 & 0.5941 & 0.6174 & 0.6860 & 0.0609 & 0.6488 & 0.2747 & & & \\
\hline
\end{tabular}

Table 5. Descriptive statistics for the real data.

\begin{tabular}{cccc}
\hline Measure & Value & Measure & Value \\
\hline$n$ & 107 & Minimum & 0.0168 \\
Maximum & 0.8781 & Mean & 0.468851 \\
$Q_{1}$ & 0.348 & $Q_{3}$ & 0.6012 \\
Median & 0.4741 & Mean Deviation & 0.152078 \\
variance & 0.0368625 & SD & 0.191996 \\
Kurtosis & 2.68612 & Skewness & -0.335289 \\
\hline
\end{tabular}

We apply the values of negative log likelihood function (-LOG), Kolmogorov-Smirnov (K-S), P-value of (K-S), Androson-Darling ( $\left.\mathrm{A}^{*}\right)$, Cramér-Von Mises $\left(\mathrm{W}^{*}\right)$ and Watson statistics to verify which distribution better fits these data. The model selection was carried out using the AIC (Akaike information criterion), the BIC (Bayesian information criterion), the CAIC (consistent Akaike information criterion), Second Order of Akaike Information Criterion (AICc) and Hannan-Quinn Information Criterion (HQC):

$$
\begin{gathered}
\mathrm{AIC}=-2 \times l(\underline{\hat{\theta}})+2 k \\
\mathrm{AIC}_{\mathrm{C}}=\mathrm{AIC}+\frac{2 k(k+1)}{n-k-1} \\
\mathrm{BIC}=-2 \times l(\underline{\hat{\theta}})+k \times \log (n)
\end{gathered}
$$

and

$$
\mathrm{HQIC}=-2 \times l(\underline{\hat{\theta}})+2 k \log [\log (n)]
$$

where, $l(\underline{\hat{\theta}})$ denotes the log likelihood function evaluated at the maximum likelihood estimates, $\mathrm{k}$ is the number of parameters and $\mathrm{m}$ is the sample size. The 
MLEs, AIC, BIC, AICc and HQIC for the parameters of $\operatorname{WE}-\mathrm{G}(\alpha, \beta, \lambda, \sigma, n=1)$, TWE-G $(\alpha, \beta, \lambda, \sigma, n=2)$, GWE-G $(\alpha, \beta, \lambda, \sigma, n=6)$ and

$\operatorname{GWE}-\mathrm{G}(\alpha, \beta, \lambda, \sigma, n=7)$ are given in Table 6. Table 7 provides the values of negative log likelihood function (-LOG), Kolmogorov-Smirnov (K-S), P-value of (K-S), Androson-Darling $\left(\mathrm{A}^{*}\right)$, Cramér-Von Mises $\left(\mathrm{W}^{*}\right)$ and Watson statistics.

It is evident from Table 6, Table 7 that, the GWE-G when $(n=7)$ distribution has the lowest statistics among all fitted model. Hence, this distribution can be chosen as the best model for fitting this data set.

Figure 3(a): the plots of estimated density function of the real data set. Figure 3(b): the plots of empirical distribution and estimated CDF for the real data set.

Table 6. MLEs, AIC, BIC, HQIC, and AIC $_{C}$ for data set.

\begin{tabular}{ccccccccc}
\hline \multicolumn{1}{c}{$2^{*}$ Model } & \multicolumn{4}{c}{ MLE estimate } & \multicolumn{5}{c}{ Statistics } \\
\hline & $\hat{\alpha}$ & $\hat{\beta}$ & $\hat{\lambda}$ & $\hat{\sigma}$ & AIC & BIC & HQIC & $A I C_{C}$ \\
\hline WE-G $(n=1)$ & 10.1633 & 1.22375 & 3.82133 & 0.139725 & -49.411 & -38.7198 & -45.077 & -41.0189 \\
TWE-G $(n=2)$ & 1.45782 & 1.05258 & 4.60484 & 0.036615 & -50.286 & -39.595 & -45.952 & -41.8941 \\
GWE-G $(n=6)$ & 0.01618 & 1.42704 & 8.39064 & 0.000203 & -50.337 & -39.6458 & -46.003 & -49.9449 \\
GWE-G $(n=7)$ & 0.07822 & 1.25358 & 8.69878 & 0.000113 & -50.346 & -39.655 & -46.012 & -49.9541 \\
\hline
\end{tabular}

Table 7. The statistics -LOG, K-S, WT, $\mathrm{A}^{*}$ and $\mathrm{W}^{*}$ for data set.

\begin{tabular}{ccccccc}
\hline model & K-S & P-value & $\mathrm{A}^{*}$ & $\mathrm{~W}^{*}$ & WT & -LOG \\
\hline WE-G $(n=1)$ & 0.0591377 & 0.848387 & 0.327242 & 0.132836 & 0.132358 & -28.7055 \\
TWE-G $(n=2)$ & 0.0508221 & 0.945088 & 0.237994 & 0.117374 & 0.117362 & -29.1431 \\
GWE-G $(n=6)$ & 0.048713 & 0.961402 & 0.225738 & 0.114653 & 0.114609 & -29.1685 \\
GWE-G $(n=7)$ & 0.047872 & 0.966937 & 0.220327 & 0.113465 & 0.113409 & -29.1732 \\
\hline
\end{tabular}

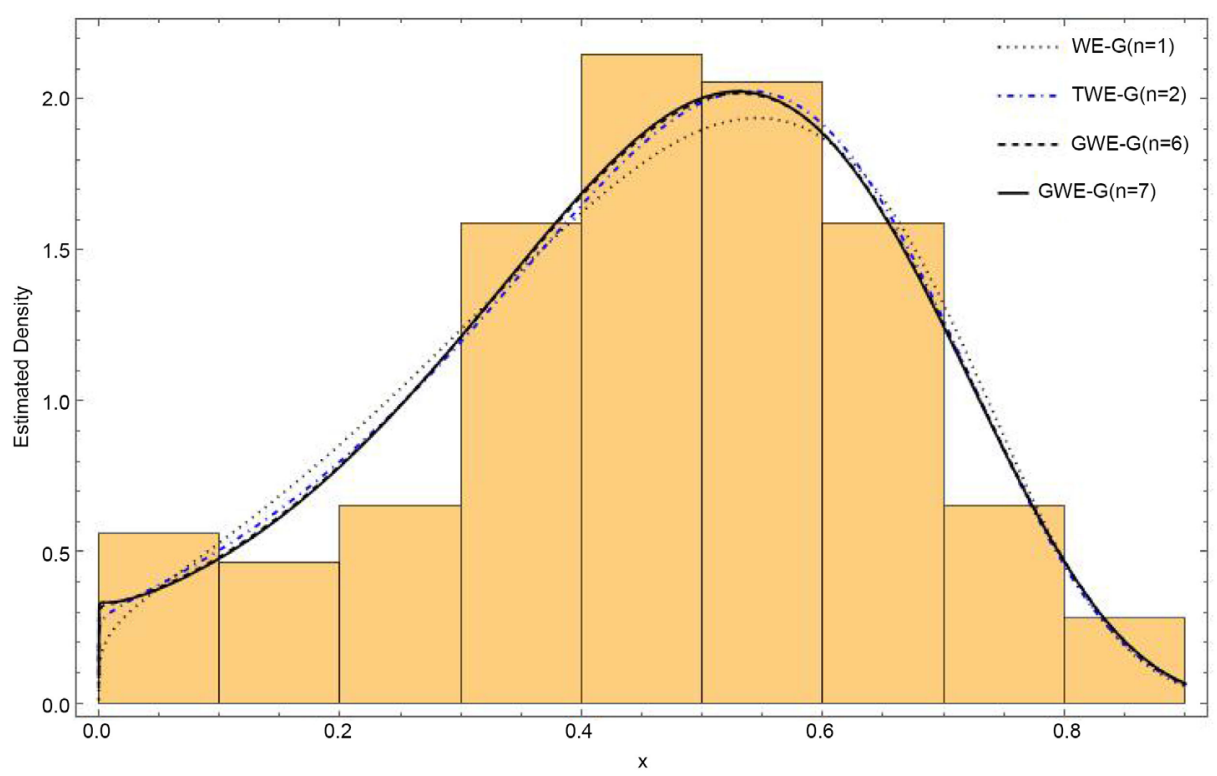

(a) 


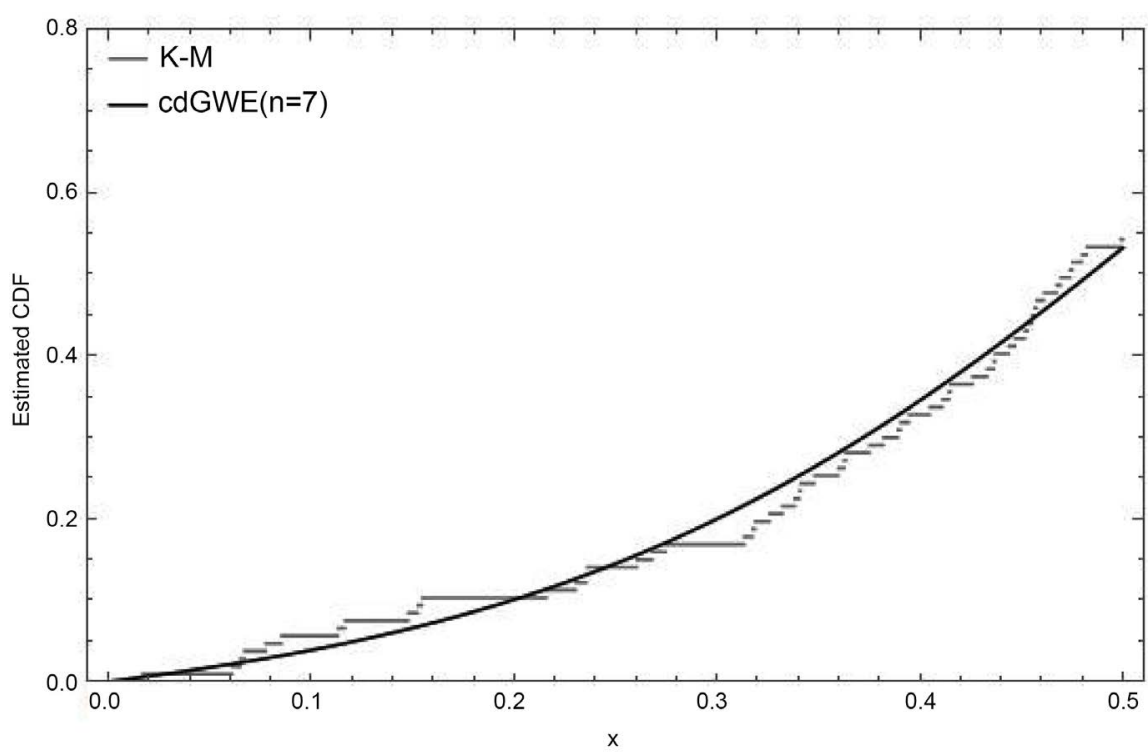

(b)

Figure 3. (a) Estimated density for real data set; (b) Estimated CDF for real data set.

\section{Conclusion}

In this paper, we discuss a new extension version of the Gompertz distribution generated by integral transform of the PDF of the generalized weighted exponential distribution. Statistical properties of the GWE-G are viewed. Maximum likelihood estimators of the GWE-G parameters are obtained. Moreover, the new model with its sub-models is fitted to real data set and it is shown that this model has a better performance among the compared distributions.

\section{Conflicts of Interest}

The authors declare no conflicts of interest regarding the publication of this paper.

\section{References}

[1] Eugene, N., Lee, C. and Famoye, F. (2002) Beta-Normal Distribution and Its Applications. Communications in Statistics-Theory and Methods, 31, 497-512. https://doi.org/10.1081/STA-120003130

[2] Zografos, K. and Balakrishnan, N. (2009) On Families of Beta- and Generalized Gamma-Generated Distributions and Associated Inference. Statistical Methodology, 6, 344-362. https://doi.org/10.1016/j.stamet.2008.12.003

[3] Amini, M., MirMostafaee, S.M.T.K. and Ahmadi, J. (2014) Log-Gamma-Generated Families of Distributions. Statistics, 48, 913-932. https://doi.org/10.1080/02331888.2012.748775

[4] Cordeiro, G.M. and de Castro, M. (2011) A New Family of Generalized Distributions. Journal of Statistical Computation and Simulation, 81, 883-893. https://doi.org/10.1080/00949650903530745

[5] Alexander, C., Cordeiro, G.M., Ortega, E.M.M. and Sarabia, J.M. (2012) Generalized Beta Generated Distributions. Computational Statistics and Data Analysis, 56, 
1880-1897. https://doi.org/10.1016/j.csda.2011.11.015

[6] Ristic, M.M. and Balakrishnan, N. (2012) The Gamma-Exponentiated Exponential Distribution. Journal of Statistical Computation and Simulation, 82, 1191-1206. https://doi.org/10.1080/00949655.2011.574633

[7] Cordeiro, G.M., Ortega, E.M.M. and da Cunha, D.C.C. (2013) The Exponentiated Generalized Class of Distributions. Journal of Data Science, 11, 1-27.

[8] Alzaatreh, A., Lee, C. and Famoye, F. (2013) A New Method for Generating Families of Continuous Distributions. Metron, 71, 63-79.

https://doi.org/10.1007/s40300-013-0007-y

[9] Bourguignon, M., Silva, R.B. and Cordeiro, G.M. (2014) The Weibull-G Family of Probability Distributions. Journal of Data Science, 12, 53-68.

[10] Cordeiro, G.M., Hashimoto, E.M. and Ortega, E.M.M. (2014) The McDonald Weibull Model. Statistics. A Journal of Theoretical and Applied Statistics, 48, 256-278. https://doi.org/10.1080/02331888.2012.748769

[11] Alizadeh, M., Emadi, M., Doostparast, M., Cordeiro, G.M., Ortega, E.M.M. and Pescim, R.R. (2015) A New Family of Distributions: The Kumaraswamy Odd Log-Logistic, Properties and Applications. Hacettepe Journal of Mathematics and Statistics, 44, 1491-1512.

[12] Tahir, M.H., Cordeiro, G.M., Alzaatreh, A., Mansoor, M. and Zubair, M. (2016) The Logistic-X Family of Distributions and Its Applications. Communications in Statistics-Theory and Methods, 45, 7326-7349. https://doi.org/10.1080/03610926.2014.980516

[13] Abd El-Bar, A.M.T. and Ragab, I.E. (2019) On Weighted Exponential-Gompertz Distribution: Properties and Application. Journal of Taibah University for Science, 13, 616-627. https://doi.org/10.1080/16583655.2019.1600277

[14] Gupta, R.D. and Kundu, D. (2009) A New Class of Weighted Exponential Distributions. Statistics, 43, 621-634. https://doi.org/10.1080/02331880802605346

[15] Shakhatreh, M.K. (2012) A Two-Parameter of Weighted Exponential Distributions. Statistics and Probability Letters, 82, 252-261.

https://doi.org/10.1016/j.spl.2011.10.008

[16] Kharazmi, O., Mahdavi, A. and Fathizadeh, M. (2015) Generalized Weighted Exponential Distribution. Communications in Statistics-Simulation and Computation, 44, 1557-1569. https://doi.org/10.1080/03610918.2013.824092

[17] Pollard, J.H. and Valkovics, E.J. (1992) The Gompertz Distribution and Its Applications. Genus, 48, 15-28.

[18] Bakouch, H.S. and Abd El-Bar, A.M.T. (2017) A New Weighted Gompertz Distribution with Applications to Reliability Data. Applications of Mathematics, 62, 269-296. https://doi.org/10.21136/AM.2017.0277-16

[19] Kayid, M. and Ahmad, I.A. (2004) On the Mean Inactivity Time Ordering with Reliability Applications. Probability in the Engineering and Informational Sciences, 18, 395-409. https://doi.org/10.1017/S0269964804183071

[20] Kayid, M. and Izadkhah, S. (2014) Mean Inactivity Time Function, Associated Orderings, and Classes of Life Distributions. IEEE Transactions on Reliability, 63, 593-602. https://doi.org/10.1109/TR.2014.2315954

[21] Brito, R.S. (2009) Estudo de Expansões Assintóticas, Avaliação Numérica de Momentos das Distribuições Beta Generalizadas, Aplicações em Modelos de Regressão e Análise Discriminante. Master-s thesis, Universidade Federal Rural de Pernambuco. 